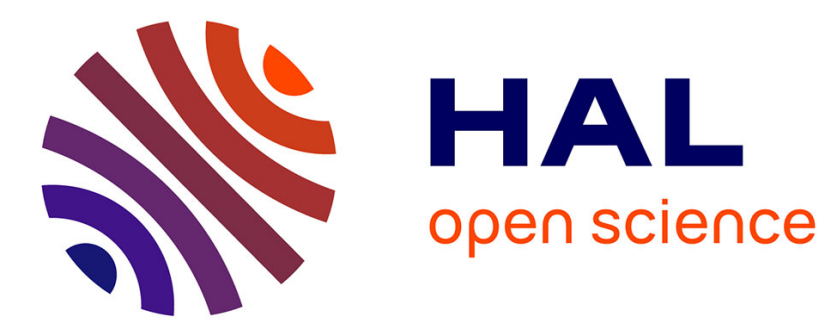

\title{
Bloch Analysis of Artificial Lines and Surfaces Exhibiting Glide Symmetry
}

Mohammad Bagheriasl, Oscar Quevedo-Teruel, Guido Valerio

\section{To cite this version:}

Mohammad Bagheriasl, Oscar Quevedo-Teruel, Guido Valerio. Bloch Analysis of Artificial Lines and Surfaces Exhibiting Glide Symmetry. IEEE Transactions on Microwave Theory and Techniques, 2019, 67 (7), pp.2618-2628. 10.1109/TMTT.2019.2916821 . hal-02505521

\section{HAL Id: hal-02505521 \\ https://hal.science/hal-02505521}

Submitted on 11 Mar 2020

HAL is a multi-disciplinary open access archive for the deposit and dissemination of scientific research documents, whether they are published or not. The documents may come from teaching and research institutions in France or abroad, or from public or private research centers.
L'archive ouverte pluridisciplinaire HAL, est destinée au dépôt et à la diffusion de documents scientifiques de niveau recherche, publiés ou non, émanant des établissements d'enseignement et de recherche français ou étrangers, des laboratoires publics ou privés. 


\title{
Bloch Analysis of Artificial Lines and Surfaces Exhibiting Glide Symmetry
}

\author{
Mohammad Bagheriasl, Oscar Quevedo-Teruel, Senior Member, IEEE, and Guido Valerio, Senior Member, IEEE
}

\begin{abstract}
Glide-symmetric structures have recently emerged as a smart choice to design planar lenses and electromagnetic band-gap materials. We discuss here under which conditions a glide-symmetric structure is equivalent to a non-glide-symmetric structure with a reduced period. To this aim, we propose an analysis method based on network theory to efficiently derive the dispersive behavior of these periodic structures. Both phase and attenuating constants can be determined, with potential applications to both guiding and radiating structures. Retaining higher-order modal interactions among cells helps to derive the dispersive behavior of periodic structures more accurately. Furthermore, we take advantage of the higher symmetry of these structures to decrease the computational cost by considering only one half or one quarter of a unit cell instead of the entire cell. We study one and two-dimensional glide-symmetric structures and confirm the validity of our analysis with comparisons from commercial software.
\end{abstract}

Index Terms-Glide symmetry, higher symmetry, transmission matrix, periodic structures, dispersion analysis.

\section{INTRODUCTION}

A RTIFICIAL materials made by periodic structures have long been used as a solution to control the electromagnetic properties of guided and radiating waves [1]-[3]. Introducing spatial higher symmetries within each unit cell provides interesting dispersive properties that make these structures excellent candidates for many applications. The higher symmetries used in electromagnetics are twist and glide. Twistsymmetric structure are invariant under a translation followed by a rotation [4]-[6], whereas glide-symmetric structures are invariant under a translation and a mirroring [7] (see Fig. 1(a)).

Glide symmetry was at first studied in connection to the theory of 1-D periodic waveguides [8]-[11]. Recently, a surge of interest in studying 2-D periodic structures with glide symmetry has started in the framework of metamaterial research [12]-[16]. They find applications such as wideband artificial lenses and electromagnetic band-gap materials. In fact, 2-D glide-symmetric metasurfaces reduce considerably the dispersion of waves propagation with respect to simple

Manuscript received September 18, 2018; revised December 12, 2018; accepted April 25, 2019. Date of publication MM XX, 2019; date of current version MM XX, 2019 .This work was supported by the French governement under the ANR grant HOLeYMETA ANR JCJC 2016 ANR-16-CE24-0030 and by Sorbonne Universités under the Emergence 2016 grant MetaSym. (Corresponding author: Mohammad Bagheriasl.)

Mohammad Bagheriasl and Guido Valerio are with Sorbonne Université, Laboratoire d'Électronique et Électromagnétisme, UR2, L2E, F75005 Paris, France (e-mail: mohammad.bagheriasl@ sorbonne-universite.fr, guido.valerio@sorbonne-universite.fr).

O. Quevedo-Teruel is with the Department of Electromagnetic Engineering, KTH Royal Institute of Technology, 11428 Stockholm, Sweden (e-mail: oscarqt@kth.se).
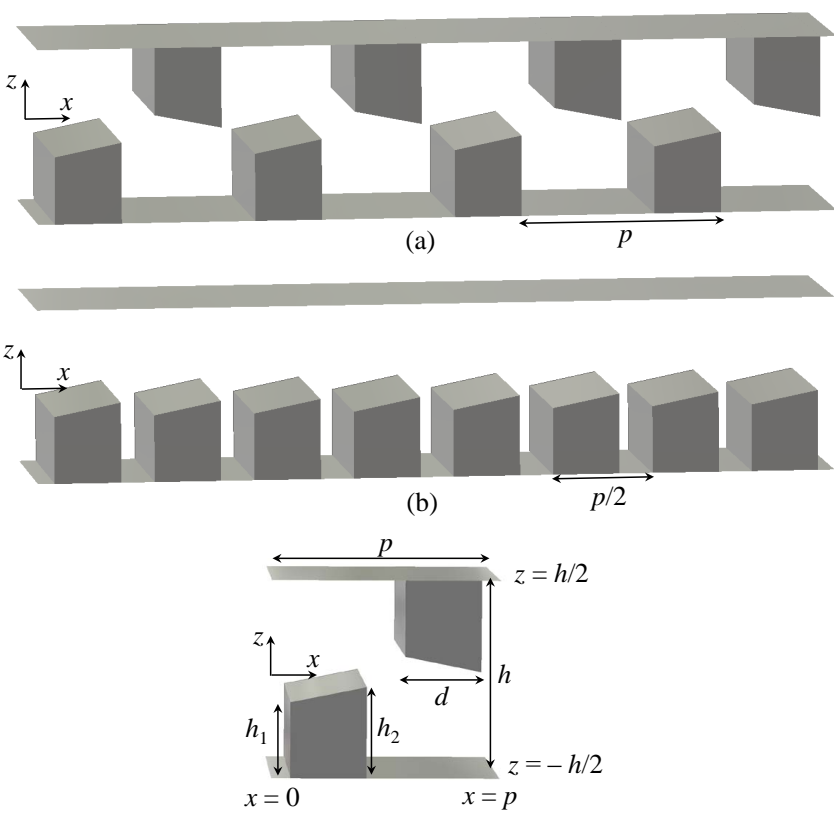

(c)

Fig. 1. (a) 1-D glide-symmetric line whose period is $p$. (b) Non-glidesymmetric periodic line obtained by moving the top metals on the bottom plate. Its period is $p / 2$. (c) Unit cell of the glide-symmetric structure.

periodic structures, mimicking an artificial material whose refractive index is stable over an ultra-wide band [17]-[19]. Furthermore, 2-D glide-symmetric metasurfaces improve both the frequency width and the attenuation of stop-bands with respect to simple periodic structures, offering new solutions for field confinement [20] and gap-waveguide technology [21], [22].

These interesting dispersive effects have recently stimulated research in order to explain the effect of glide symmetry on periodic structures, i.e. to correctly define the difference between the structures in Fig. 1(a) and (b). On one hand, mode-matching [23]-[25] has been proposed for glidesymmetric holey metasurfaces and corrugations. However, this approach does not provide a physical interpretation of the glide symmetry impact in a periodic structure. On the other hand, the simplicity of circuit-based modeling methods can lead to a better physical explanation of glide symmetry effects. For this reason, this approach was chosen in [26] and [27] for simpler 1-D glide and twist configurations, respectively. Specifically, the structure analyzed in [26] consists of two glide-symmetric corrugated structures. Under the assumption that each corrugation can be replaced by Marcuvitz's closed- 
form equivalent circuit [28], it was easily shown that the glide symmetry effect is equivalent to simply halving the spatial period. The study in [29], discussing reduced representations of several kinds of higher-symmetric periodic lines proposed in [30], has recently shown that a more correct condition for this conclusion is to neglect interactions due to localized excitation of higher-order waveguide modes.

In this work we explain the difference between glide and non-glide structures in a general framework which does not depend on their specific technology, but only on the symmetry properties of their unit cells. This is done by comparing the interaction among scatterers in glide and non-glide configurations. In doing so, we define two classes of glidesymmetric structures: reducible and irreducible. The former ones are equivalent to non-glide-symmetric structures with reduced period (halved period in 1-D configurations), as those discussed in [26]. In the latter ones, glide symmetry is not reducible to a simple period reduction. In order to explain the difference between these classes, we describe a unit cell as a multimodal equivalent network, where several modes are defined on its access ports and are associated to equivalent voltages and currents. A multimodal transmission matrix allows for performing a Bloch analysis where interactions due to the localized excitation of higher-order modes are correctly modeled, thus generalizing the Bloch analysis in [1]. The difference between reducible and irreducible structures will be proved to be the contribution of higher-order modes to the cell coupling.

Furthermore, we transform for the first time the eigenvalue problem based on the glide operator [8] to a transmissionmatrix formulation. This has several advantages. Firstly, it restricts the computational domain of the problem to one-half of the unit cell (one quarter of a suitable non-minimal cell in the 2-D case). This leads to a reduction of the computation time, accurately quantified in the paper. In addition, it shows the different effects of higher-order modes according to their parity properties, and confirms the role of modal coupling in reducible and irreducible lines. This means that future research on equivalent models of specific glide structures will have to include higher-order modal couplings according to the formulation described here. Finally, we stress that, unlike the methods used in commercial software, our method accurately computes both the phase and the attenuation constants of a Bloch mode. Therefore, it can be used to study guiding as well as open structures (such as leaky-wave antennas, where the attenuation is due to a progressive radiation of the traveling wave [31], [32]).

All these analyses are performed at first for 1-D glidesymmetric structures. The generalized eigenvalue problem is then extended for the first time to 2-D configurations leading to the first complete formulation of the problem for glidesymmetric surfaces.

The paper is organized as follows. In Sec. II, we present a multimodal transmission matrix formulation for dispersive analysis of 1-D periodic structures. We will then use it to discuss the differences between reducible and irreducible structures and show relevant validations with numerical results. In Sec. III, we transform an eigenvalue problem stated in terms of the glide-symmetric operator into a multimodal transmission-matrix formulation. This limits our computation domain to one half of a unit cell. In Sec. IV we formulate for the first time an eigenvalue problem in terms of the 2-D glide-symmetric operator and extend the previous analyses to 2-D glide-symmetric structures.

\section{Multimodal Couplings IN 1-D GLIDE-SyMMETRIC STRUCTURES}

In this section, we study the effect of multimodal interactions among unit cells, and their effect on 1-D glidesymmetric structures. Even if Floquet boundary conditions are well known, the formulation is stated explicitly for two main reasons. Floquet conditions are transformed here to a less common multimodal transmission matrix, which allows to distinguish between reducible and irreducible structures. Moreover, these boundary conditions help to highlight the difference with the formulation based on glide boundary conditions presented in Section III.

\section{A. Multimodal Periodic Boundary Conditions Along Lines}

Fig. 1 (a) shows a 1-D glide-symmetric structure composed of two corrugated metallic surfaces off-shifted with respect to each other. For simplicity the structure is assumed invariant along the $y$ direction. A 1-D glide-symmetric structure is invariant under the glide symmetry operator $G_{p \hat{\boldsymbol{x}}}$, the composition of an $x$-translation of length $p / 2$ and a $z$-reflection around the plane $z=0$ :

$$
G_{p \hat{\boldsymbol{x}}}:(x, z) \rightarrow(x+p / 2,-z)
$$

We recall that a 1-D periodic structure is invariant under the translation operator $T_{p \hat{\boldsymbol{x}}}$ of length $p$ along the direction of periodicity $x$. Since the composition of two glide operators is $T_{p \hat{\boldsymbol{x}}}\left(G_{p \hat{\boldsymbol{x}}}^{2}=T_{p \hat{\boldsymbol{x}}}\right)$, a glide-symmetric structure is also a periodic structure of period $p$. The periodic non-glidesymmetric structure in Fig 1 (b) can be obtained from the glide-symmetric structure in Fig. 1 (a) by mirroring the upper corrugations and moving them from the top to the bottom plate. Notice that doing so we have halved the period of the resulting line. Later in the section we will compare the dispersion behaviors of the two structures in Figs. 1 (a) and 1 (b), which are referred in the following as the non-glide and the glide structure.

The unit cell of the glide-symmetric line is depicted in Fig. 1 (c). It is well known that a periodic structure can be studied by limiting the analysis to its unit cell, and enforcing a Floquet boundary condition on its periodic boundaries $(x=0$ and $x=p$ in this case). This leads to an eigenvalue problem associated to the translation operator:

$$
T_{p \hat{\boldsymbol{x}}}[\boldsymbol{E}(x, z)]=\boldsymbol{E}(x+p, z)=e^{-j k_{x} p} \boldsymbol{E}(x, z)
$$

whose eigenvalue is related to the propagation constant of a Bloch mode $\left(k_{x}\right)$ and whose eigenvector is the modal field. In general, $k_{x}=\beta-j \alpha$ is a complex quantity. Its real part $\beta$ is the modal phase constant, while the opposite of its imaginary part $\alpha$ is the attenuation constant. $\alpha$ can be related either to the presence of a stop-band or to material or radiation losses along 
the periodic line [1]. The boundary condition (2) is given here in terms of the electric field $\boldsymbol{E}$, but it could be equivalently stated in terms of the magnetic field $\boldsymbol{H}$. This holds for all the boundary conditions used in the following, written for brevity only in terms of $\boldsymbol{E}$.

We will use a multimodal transmission-matrix analysis to transform (2) into a finite-dimensional problem. We describe a unit cell as a multiport network, whose two geometrical faces $(x=0$ and $x=p$ in Fig. 1 (c)) are the contact ports with adjacent cells. On each of these faces, one can define $N$ modes of the parallel-plate waveguide limited by the planes $z=-h / 2$ and $z=h / 2$. The $n$-th mode on the faces 1 and 2 is associated with voltages and currents $V_{1}^{(n)}, I_{1}^{(n)}$ and $V_{2}^{(n)}, I_{2}^{(n)}$, respectively. We then define a multimodal generalized transmission matrix (or "T matrix") $\underline{T}$ as:

$$
\begin{gathered}
{\left[\begin{array}{c}
\boldsymbol{V}_{2} \\
\boldsymbol{I}_{2}
\end{array}\right]=\underline{\boldsymbol{T}} \cdot\left[\begin{array}{c}
\boldsymbol{V}_{1} \\
\boldsymbol{I}_{1}
\end{array}\right]} \\
\text { where } \boldsymbol{V}_{i}=\left(\begin{array}{c}
V_{i}^{(1)} \\
V_{i}^{(2)} \\
\vdots \\
V_{i}^{(N)}
\end{array}\right) \text { and } \boldsymbol{I}_{i}=\left(\begin{array}{c}
I_{i}^{(1)} \\
I_{i}^{(2)} \\
\vdots \\
I_{i}^{(N)}
\end{array}\right)
\end{gathered}
$$

relating the voltage and current values on the face 2 to those on the face 1. The possible use in (3) of $N>1$ modes on each geometrical face of the unit cell is the main difference with respect to usual monomodal $(N=1) A B C D$ transmission matrices [1]. We will show in Sec. II-B that higher-order modes can prove necessary to extract the accurate dispersive behavior of glide-symmetric structures with irreducible cells. This will improve the accuracy of the results by avoiding the use of multi-cell analysis as done in [33]. In order to solve for the dispersion relation of the structure, we apply the Floquet conditions (2) at the boundaries of the unit cell in terms of voltages and currents:

$$
\left[\begin{array}{l}
\boldsymbol{V}_{2} \\
\boldsymbol{I}_{2}
\end{array}\right]=\mathrm{e}^{-j k_{x} p}\left[\begin{array}{l}
\boldsymbol{V}_{1} \\
\boldsymbol{I}_{1}
\end{array}\right]
$$

Solving the eigenvalue problem (4) for different frequencies finds $k_{x}$ at these frequencies. In the next subsections, we will use this eigenvalue problem to extract the dispersive relations of the 1-D glide-symmetric structure of Fig. 1 (a). We use a commercial software (frequency domain solver in CST Microwave Studio [34]) to obtain the impedance matrix of the unit cell. Then, this matrix is easily converted to the transmission matrix $\underline{\boldsymbol{T}}$ that we can use in (3). After solving for the dispersion relation, we compare the dispersion results of our proposed Bloch mode analysis with the results directly obtained from the eigensolver analysis of the CST Microwave Studio. This will validate our proposed method by verifying its accuracy. It is good to note that the imaginary part $\alpha$ of the wavenumber cannot be compared to the CST eigensolver results as the latter software does not compute this quantity.

\section{B. Reducible and Irreducible Glide Structures}

In [26], an equivalence between the structures in Fig. 1 (a) and Fig. 1 (b) is stated, based on the validity of an equivalent circuit there proposed. This equivalent circuit neglects the higher-order modes at the two geometrical faces $x=0$ and $x=p$. It corresponds then to a formulation of problem (3) with $N=1$, where only one mode is retained on each unitcell face. In [26], this assumption is shown to be valid as long as $d>p / 2$. In [29], the validity is more correctly stated in terms of higher-mode relevance. In this sub-section, we class glide-symmetric structures in two kinds, and relate this classification with the nature of the modal interaction between adjacent cells. Note that localized higher-order modal interactions in 1-D periodic structures is known to be relevant, for example leading to the presence of lumped elements in equivalent circuits [35]. We explore here the impact of this phenomenon on glide-symmetric lines.

In reducible glide-symmetric structures, the mirroring operation does not have an impact on the dispersive diagram. For instance, a reducible line as in Fig. 1 (a) has the same dispersion relation as the structure in Fig. 1 (b). Conversely, in irreducible glide-symmetric structures the mirroring operation does have an impact, so that an irreducible line as in Fig. 1 (a) has a different dispersion relation from the structure in Fig. 1 (b). As shown in the following analyses, the same geometry can be reducible or irreducible according to the values of its geometrical and physical parameters and the frequency range of interest.

Using the T-matrix analysis, we aim at demonstrating that the difference between reducible and irreducible structures lies in the impact of higher-order modes on each unit-cell boundary. Reducible structures require only one mode at each face, whereas irreducible lines (or their non-glide periodic lines) need higher-order modes at each face to compute the dispersion diagram correctly.

Fig. 2 (a) shows the dispersion curves for the structures in Fig. 1 (a) and (b), whose geometrical parameters are $p=3$ $\mathrm{mm}, d=0.25 \mathrm{~mm}, h_{1}=0.35 \mathrm{~mm}, h_{2}=0.45 \mathrm{~mm}$ and $h=1$ $\mathrm{mm}$. The results are obtained with the CST eigensolver. This corresponds to a reducible glide symmetry, since the glide and non-glide dispersion diagrams are superimposed. In Fig. 2 (b), we plot the diagrams obtained with the multimodal T-matrix method. It is clear that using higher-order port modes does not change the results as the first mode is already enough to reach the accurate dispersion diagram. Considering the full-wave CST eigensolver result as our reference, we take the relative error of the frequency as a criterion for the convergence of the method. Therefore, the definition of the convergence is:

$$
\left|\frac{f-f_{\text {full-wave }}}{f_{\text {full-wave }}}\right|<\delta
$$

At each phase constant, $f$ is the frequency given by the transmission-matrix method, $f_{\text {full-wave }}$ is the frequency given by CST and $\delta$ is the relative error which defines the criteria for convergence. We use a $\delta=0.02$ for all the results given in the paper. For instance, in the previous result, the convergence inequality given in eq. (5) holds with $N \geq 1$. Therefore, the convergence occurs at $N=1$ mode. This suggests that when the adjacent corrugations are not very close to each other, they are reducible and the mirroring operation in the glide symmetry has no impact on the dispersion relation. Therefore, 


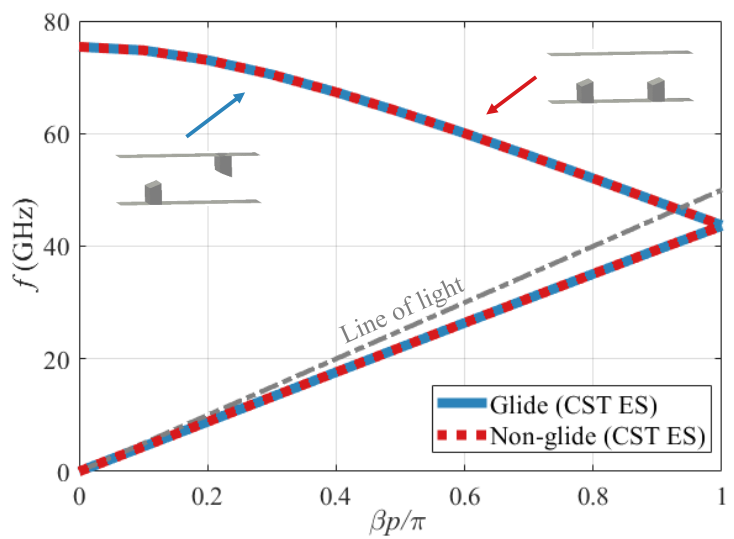

(a)

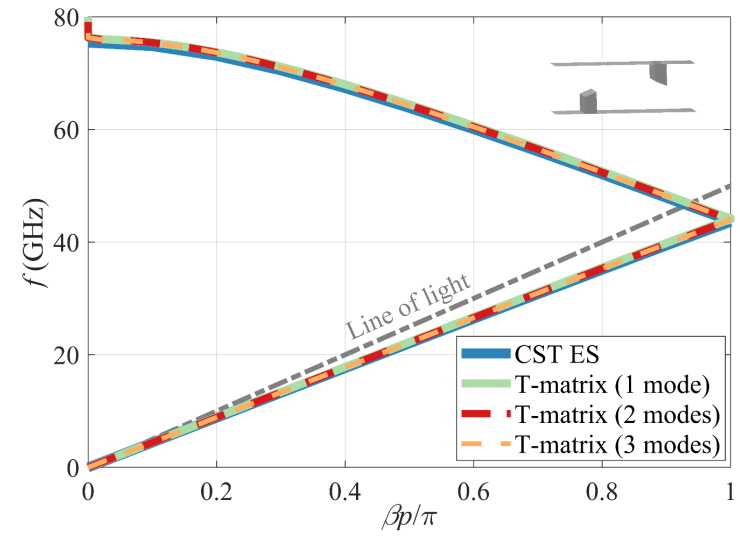

(b)

Fig. 2. Dispersion diagram of the structures in Fig. 1 (a) and (b) with geometrical parameter: $p=3 \mathrm{~mm}, d=0.25 \mathrm{~mm}, h_{1}=0.35 \mathrm{~mm}, h_{2}=0.45$ $\mathrm{mm}$ and $h=1 \mathrm{~mm}$.

the presence of glide symmetry is strictly equivalent to a reduction of the period of a factor two. This will be confirmed by the formulation using glide boundary conditions proposed in Section III.

To study an irreducible glide-symmetric periodic cell, we consider to shorten the period and increase the width of the metals to the values $p=1.5 \mathrm{~mm}$ and $d=0.5 \mathrm{~mm}$. In this case, the proximity of the scatterers is expected to define an irreducible structure. Fig. 3 (a) depicts the dispersion diagram of the glide structure and its non-glide counterpart with halved period, obtained with the CST eigensolver. Unlike the reducible example, the dispersion diagrams of these two topologies are totally different.

Furthermore, in Fig. 3 (b), we show the results for the glidesymmetric structure obtained with the multimodal $\mathrm{T}$ matrix for different number of port modes. Retaining only one waveguide mode results in a wrong dispersion diagram, with an incorrect stop band at mid-frequencies. In contrast, the inclusion of one higher-order mode already leads to the correct dispersion diagram of the periodic structure (convergence of the results for $N=2$ ). The curve with $N=3$ once again exhibits the robustness of the method: where considering a higher number of modes than necessary for convergence, the converged results do not change. The different symmetry of modes 1 and

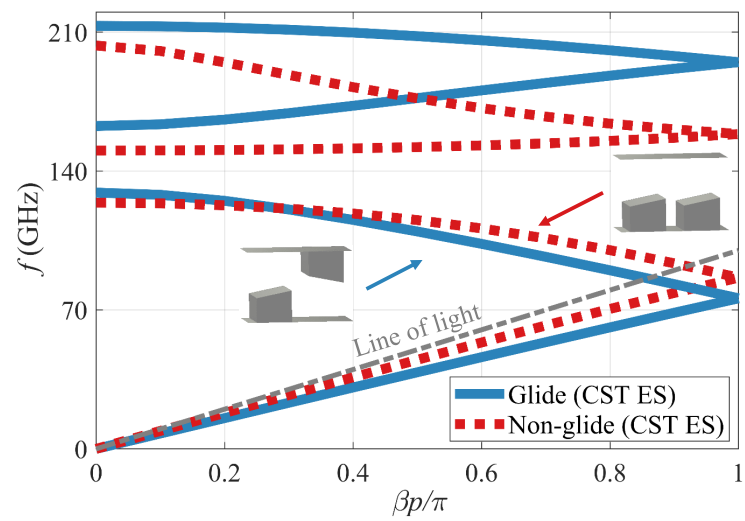

(a)

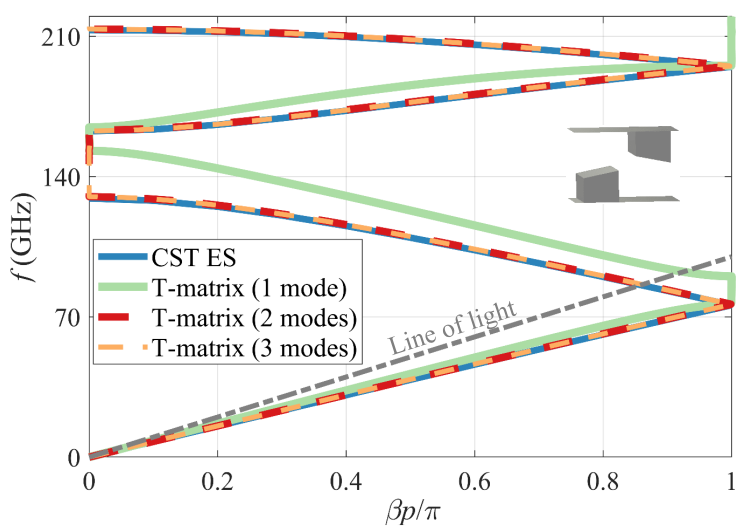

(b)

Fig. 3. Dispersion diagram of the structures in Fig. 1 (a) and (b) with parameter values: $p=1.5 \mathrm{~mm}, d=0.5 \mathrm{~mm}, h_{1}=0.35 \mathrm{~mm}, h_{2}=0.45$ $\mathrm{mm}$ and $h=1 \mathrm{~mm}$.

2 with respect to $z=0$ plane suggests that the mirrored nature of half-cells in glide-symmetries treats modes with different types of symmetry differently. In the next section, we use this observation to suggest a modified analysis that uses the higher symmetry aspect of glide-symmetric structures to obtain the dispersion relation from half of a unit cell.

\section{1-D GLIDE-SYMMETRIC CONDITIONS ON THE BOUNDARIES OF HALF A CELL}

In this section, we exploit glide symmetry in order to limit the computational domain to a sub-region of the entire unit cell. A generalized Floquet theorem discussed in [8] shows that the study of a glide-symmetric line can be limited to half of its unit cell (the region $0<x<p / 2$ in Fig. 1 (c)) by formulating the following eigenvalue problem associated to the glide operator:

$$
G_{p \hat{\boldsymbol{x}}}[\boldsymbol{E}(x, z)]=\boldsymbol{E}(x+p / 2,-z)=e^{-j k_{x} p / 2} \boldsymbol{E}(x, z)
$$

This means that the field measured after a translation of half a period and a mirroring with respect to the glide plane is a scalar multiple of the field at the initial position. It is easy to realize that two $G_{p} \hat{\boldsymbol{x}}$ operations yield the eigenvalue problem (2). 
It is important to remark that a minus sign could be added to the last term of (6) by keeping the consistency with (2). However, this sign would not change the result of the problem. In fact, if a - sign is introduced, the $k_{x}$ wavenumber is transformed into the $k_{x}^{\prime}=k_{x}+2 \pi / p$ wavenumber. Since $k_{x}^{\prime}$ and $k_{x}$ differ of $2 \pi / p$, they are simply two harmonics of the same Bloch mode [23]. This issue is slightly different in 2-D configurations and will be discussed in the next section.

We want to formulate the glide eigenvalue problem (6) by means of a multimodal transmission matrix. To this aim, we define the multimodal transmission matrix $\underline{\boldsymbol{T}}_{1 / 2}$ associated to one half of a unit cell $(0<x<p / 2$ in Fig. 1 (c)). However, while a transmission matrix performs a translation along the line, here we need a glide operator, consisting of a translation and a mirroring. We overcome this issue by writing the glide boundary condition (6) for each mode defined at the geometrical ports of the half cell. Each mode is either odd or even with respect to the mirroring direction $z$, and it is here named $e_{z}^{\text {even }}$ and $e_{z}^{\text {odd }}$ respectively. Due to the bidimensional nature of the problem, we consider here the $z$ components of modal electric fields, used to define equivalent voltages at each port. The same symmetry holds for the $x$ components of the modal magnetic fields, which define equivalent currents. For both these components, the mirror operation reduces then to an additional product by either $\mathrm{a}+1$ or $\mathrm{a}-1$ factor:

$$
\left\{\begin{array}{lll}
e_{z}^{\text {even }}(x,-z) & =e_{z}^{\text {even }}(x, z) \\
e_{z}^{\text {odd }}(x,-z) & =-e_{z}^{\text {odd }}(x, z)
\end{array}\right.
$$

Therefore, the translation of half a cell of each mode is

$$
\left\{\begin{array}{l}
T_{\hat{\boldsymbol{x}} p / 2} \boldsymbol{E}^{\text {even }}=e^{-j k_{x} p / 2} \boldsymbol{E}^{\text {even }} \\
T_{\hat{\boldsymbol{x}} p / 2} \boldsymbol{E}^{\text {odd }}=-e^{-j k_{x} p / 2} \boldsymbol{E}^{\text {odd }}
\end{array}\right.
$$

It is easy to realize that the different sign in the translation $T_{\hat{\boldsymbol{x}} p / 2}$ in (8) according to the parity of the mode compensates the different reflection of each mode in (7). The applications of the two transformations (8) and (7) is equivalent to a glide operation, and it is consistent with the glide boundary condition (6) for both even and odd modes. Finally, if a voltage and a current are associated to each mode, the boundary conditions (8) for each voltage and current can be stated as

$$
\left[\begin{array}{c}
V_{2}^{(i)} \\
I_{2}^{(i)}
\end{array}\right]=q^{(i)} \cdot e^{-j k_{x} p / 2}\left[\begin{array}{l}
V_{1}^{(i)} \\
I_{1}^{(i)}
\end{array}\right]
$$

where $q^{(i)}=\left\{\begin{array}{cl}+1 & \text { mode } i \text { has even symmetry } \\ -1 & \text { mode } i \text { has odd symmetry }\end{array}\right.$

We can write this condition in matrix form by defining a sign matrix $\underline{Q}$ whose $i$ th diagonal element is equal to $q^{(i)}$ in (9).

$$
\underline{\boldsymbol{Q}}=\left[\begin{array}{cccc}
q^{(1)} & 0 & \cdots & 0 \\
0 & q^{(2)} & \ddots & \vdots \\
\vdots & \ddots & \ddots & 0 \\
0 & \cdots & 0 & q^{(N)}
\end{array}\right]
$$

The glide problem becomes then

$$
\boldsymbol{T}_{1 / 2} \cdot\left[\begin{array}{l}
\boldsymbol{V}_{1} \\
\boldsymbol{I}_{1}
\end{array}\right]=e^{-j k_{x} p / 2}\left[\begin{array}{ll}
\underline{\boldsymbol{Q}} & \underline{\mathbf{0}} \\
\underline{\mathbf{0}} & \underline{\boldsymbol{Q}}
\end{array}\right] \cdot\left[\begin{array}{l}
\boldsymbol{V}_{1} \\
\boldsymbol{I}_{1}
\end{array}\right]
$$

$\underline{\mathbf{0}}$ being the $N \times N$ null matrix. For example, in a simple case where only the first two modes with different parity (TEM mode with even parity, and $\mathrm{TM}_{01}$ mode with odd parity) are retained, (10) reads as

$$
\underline{\boldsymbol{T}}_{1 / 2} \cdot\left[\begin{array}{c}
V_{1}^{(1)} \\
V_{1}^{(2)} \\
I_{1}^{(1)} \\
I_{1}^{(2)}
\end{array}\right]=e^{-j k_{x} p / 2}\left[\begin{array}{c}
V_{1}^{(1)} \\
-V_{1}^{(2)} \\
I_{1}^{(1)} \\
-I_{1}^{(2)}
\end{array}\right]
$$

With this equation, the dispersion relation of a glidesymmetric structure can be solved by considering the network parameters of only half of its unit cell. On one hand, from (10) and (11), it is clear that in reducible lines, where only one mode is retained, the glide-symmetric problem is equivalent to a periodic problem whose period is divided by two $(p / 2$ instead of $p$ ). On the other hand, in irreducible lines, the presence of higher-order modes with different parity along the mirroring direction is responsible of a different interaction between elements with respect to the non-glide periodic line.

Fig. 4, shows the dispersion diagram extracted from half of the unit cell of the irreducible glide-symmetric structure discussed in Sec. II-B. In Fig. 4(a) the phase constant $\beta$ is compared to the solution given by the eigensolver of CST. The dispersion diagram is fully recovered by considering only half of the unit cell and two port modes. The perfect agreement between results obtained with two and three modes at each face validates the convergence of the solution. Fig. 4(b) plots the normalized attenuation constant of the Bloch wave $\left(\alpha / k_{0}\right)$ with respect to frequency. As in the analysis with a full unit cell, a convergence of the method is already observed by retaining two modes. Note that the $\alpha$ in the stop-band cannot be computed with the CST eigensolver tool.

\section{2-D GLIDE-SYMMETRIC SURFACES}

In this section the previous analysis of 1-D glide-symmetric structures is generalized to 2-D configurations, i.e. artificial surfaces exhibiting glide symmetry. The two axes of periodicity are assumed orthogonal, but the formulation of the problems and all the results hold in the case of general skewed axes.

\section{A. Multimodal Periodic Boundary Conditions Along Surfaces}

Fig. 5 (a) shows a 2-D glide-symmetric structure, invariant with respect to the 2-D glide symmetry operator $G_{p_{x} \hat{\boldsymbol{x}}, p_{y} \hat{\boldsymbol{y}}}$ :

$$
G_{p_{x} \hat{\boldsymbol{x}}, p_{y} \hat{\boldsymbol{y}}}:(x, y, z) \rightarrow\left(x+p_{x} / 2, y+p_{y} / 2,-z\right)
$$

As in the 1-D case, glide symmetry implies periodicity, since $G_{p_{x} \hat{\boldsymbol{x}}, p_{y} \hat{\boldsymbol{y}}}^{2}=T_{p_{x} \hat{\boldsymbol{x}}} T_{p_{y} \hat{\boldsymbol{y}}}$. As in the 1-D case, the non glidesymmetric periodic structure in Fig 5 (b) can be obtained from the glide-symmetric structure in Fig. 5 (a) by mirroring the upper pins and moving them from the top to the bottom plate. If $p_{x}=p_{y}$, this transformation yields a periodic structure whose periods are $p / \sqrt{2}$ along $45^{\circ}$ rotated $x^{\prime}, y^{\prime}$ axes. If $p_{x} \neq p_{y}$, the formulation still applies, but a different rotation is required and different period values are obtained. The glidesymmetric unit cell is shown in Fig. 6, where black and white rectangles represent bottom and top vertical pins. 


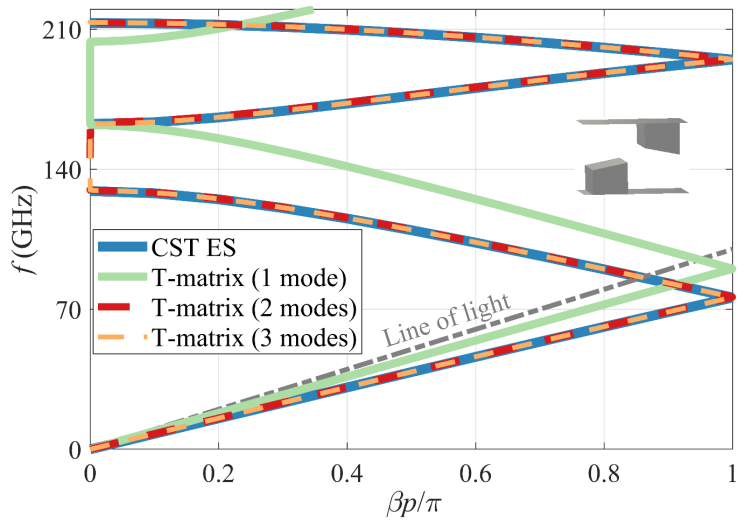

(a)

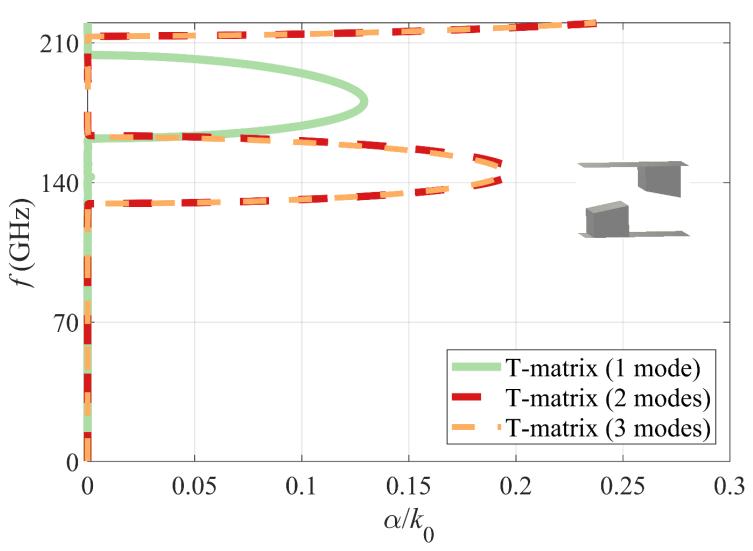

(b)

Fig. 4. Dispersion diagram of the glide-symmetric structure in Fig. 1(a) obtained from the simulation of half of a cell with $N$ modes on each geometrical face. Geometrical parameters: $p=1.5 \mathrm{~mm}, d=0.5 \mathrm{~mm}$, $h_{1}=0.35 \mathrm{~mm}, h_{2}=0.45 \mathrm{~mm}$ and $h=1 \mathrm{~mm} . N=1$ mode (green line), $N=2$ modes (dashed red line), $N=3$ modes (dashed orange line). (a) Normalized phase constant $\beta p / \pi$ vs. frequency. CST comparison (blue line), line of light dashed dotted gray line. (b) Normalized attenuation constant $\alpha / k_{0}$ vs. frequency.

The eigenvalue condition in a $2-\mathrm{D}$ periodic structure as Fig 5 (a) is:

$$
\begin{gathered}
T_{m p_{x} \hat{\boldsymbol{x}}} T_{n p_{y} \hat{\boldsymbol{y}}}[\boldsymbol{E}(x, y, z)]=\boldsymbol{E}\left(x+m p_{x}, y+n p_{y}, z\right) \\
=e^{-j\left(m k_{x} p_{x}+n k_{y} p_{y}\right)} \boldsymbol{E}(x, y, z)
\end{gathered}
$$

$m, n$ being arbitrary integers. The choice of $m=0, n=1$ and $m=1, n=0$ enforces two independent conditions covering the full boundaries of a single minimal unit cell shown in Fig. 6 (in solid line):

$$
\begin{aligned}
& \boldsymbol{E}\left(x+p_{x}, y, z\right)=e^{-j k_{x} p_{x}} \boldsymbol{E}(x, y, z) \\
& \boldsymbol{E}\left(x, y+p_{y}, z\right)=e^{-j k_{y} p_{y}} \boldsymbol{E}(x, y, z)
\end{aligned}
$$

In order to transform the eigenvalue problem (14) into a finite-dimensional problem, we need to define four geometrical faces of the unit cell, i.e., the four straight boundaries of the unit cell in Fig. 6. On each geometrical face, we can define $N$ modes as in the 1-D case. The unit cell is then equivalent to a $4 N$-port network, which can be described by a transmission matrix:

$$
\left[\begin{array}{c}
\boldsymbol{V}_{3} \\
\boldsymbol{V}_{4} \\
\boldsymbol{I}_{3} \\
\boldsymbol{I}_{4}
\end{array}\right]=\underline{\boldsymbol{T}} \cdot\left[\begin{array}{c}
\boldsymbol{V}_{1} \\
\boldsymbol{V}_{2} \\
\boldsymbol{I}_{1} \\
\boldsymbol{I}_{2}
\end{array}\right]
$$

where $\boldsymbol{V}_{i}$ and $\boldsymbol{I}_{i}(i=1, \ldots, 4)$ are vector voltages and currents as in (3). Floquet conditions can be written in terms of voltages and currents, and replaced in (15). This results in the following eigenvalue problem:

$$
\underline{\boldsymbol{T}} \cdot\left[\begin{array}{l}
\boldsymbol{V}_{1} \\
\boldsymbol{V}_{2} \\
\boldsymbol{I}_{1} \\
\boldsymbol{I}_{2}
\end{array}\right]=\left[\begin{array}{l}
e^{-j k_{x} p_{x}} \boldsymbol{V}_{1} \\
e^{-j k_{y} p_{y}} \boldsymbol{V}_{2} \\
e^{-j k_{x} p_{x}} \boldsymbol{I}_{1} \\
e^{-j k_{y} p_{y}} \boldsymbol{I}_{2}
\end{array}\right]
$$

Again, finding the roots of the determinant of the matrix on the left side of (16) provides the dispersion relation of the structure. In this section, we only consider an irreducible structure of the kind presented in Fig. 5.

Also in 2-D artificial surfaces, inter-cell interactions due to higher-order modes have been shown to be important. They are usually modeled as lumped elements in equivalent circuits [36], [37]. To demonstrate the relevance of higher-order modes in 2-D glide-symmetric structures, we apply this method to a single unit cell of an irreducible glide-symmetric structure. The parameters for this case are $h=1.15 \mathrm{~mm}, h_{1}=1$ $\mathrm{mm}, p=1.2 \mathrm{~mm}$ and $d=0.5 \mathrm{~mm}$. Fig. 7 (a) plots the dispersion diagram of the glide-symmetric unit cell next to its non-glide symmetric unit cell to confirm the irreducibility of the chosen structure. Fig. 7 (b) depicts the dispersion diagram of this glide-symmetric unit cell when propagation

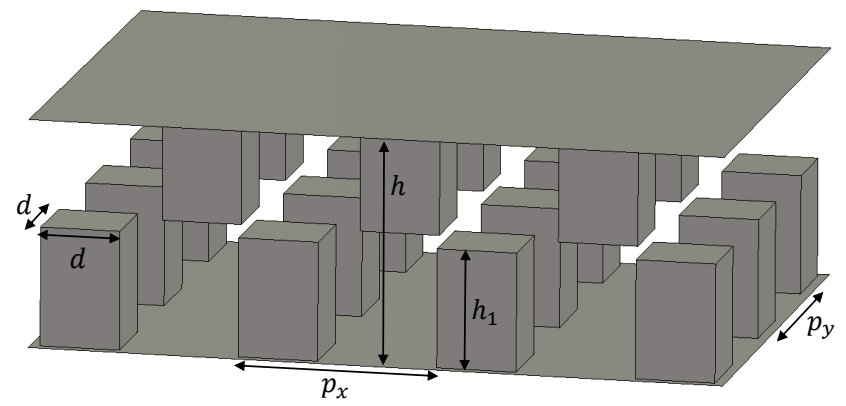

(a)

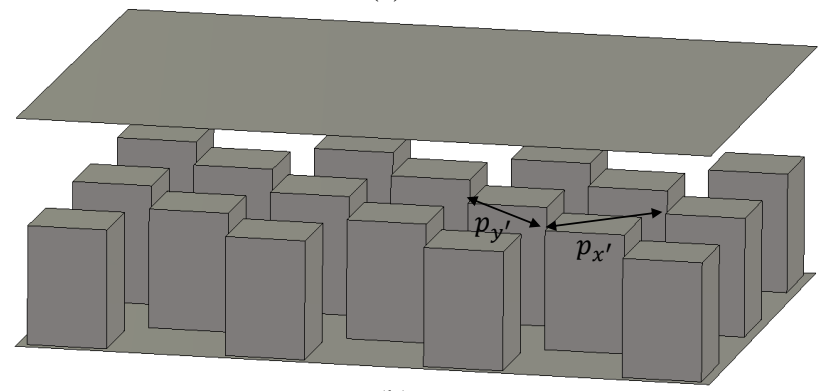

(b)

Fig. 5. (a) 2-D glide-symmetric surfaces whose periods are $p_{x}$ and $p_{y}$. (b) Non-glide-symmetric periodic surfaces obtained by moving the top pins to the bottom plate. If $p_{x}=p_{y}$ its periods along the rotated axes are $p_{x^{\prime}}=p_{x} / \sqrt{2}$ and $p_{y^{\prime}}=p_{y} / \sqrt{2}$. 


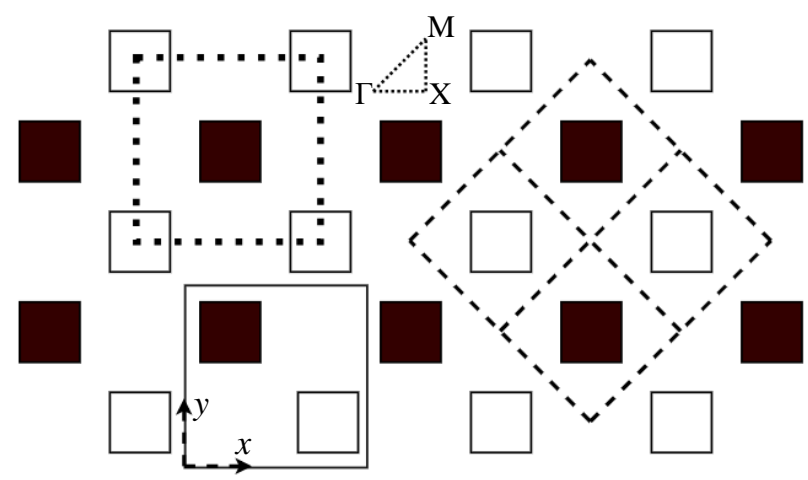

Fig. 6. Top view of the periodic structure in Fig. 5(a). Black and white squares are bottom and top pins, respectively. At the bottom left, the minimal unit cell $\left(0<x<p_{x}, 0<y<p_{y}\right)$ used in (14) is shown (solid lines). The centrally symmetric minimal unit cell is shown at the top left (dotted lines). At the right, the non-minimal unit cell and its quarter used in (18) are shown (dashed lines).

occurs along the $x$ direction while $k_{y}=0$. The results obtained from the transmission-matrix method are compared to those of the CST eigensolver for validation. This figure emphasizes the fact that a transmission-matrix with one mode on each face $(N=1)$ does not give correct results, while 11 modes are needed to obtain a perfect convergence of the result to the CST eigensolver. Fig. 7 (c) plots the normalized attenuation constant of the Bloch wave $\left(\alpha_{x} / k_{0}\right)$ with respect to frequency. This last result cannot be compared with CST, but the agreement between results with 11 and 12 modes on each face confirm the convergence of the method adding extra modes does not change the converged result. These diagrams show that the periodic structure is indeed irreducible according to the definition given in previous section, as higher-order mode interactions are relevant to obtain an accurate result. Fig. 8 sketches the full 2-D dispersion diagram using the transmission-matrix analysis with 11 modes and compares it to the CST eigensolver solution. The Brillouin diagram is here computed on the edge of an irreducible zone of the reciprocal $k$ space. Thanks to the central symmetry of a unit cell (see Ch. 2 of [2]), this region is delimited by the points $\Gamma, \mathrm{X}, \mathrm{M}$ as shown in Fig. 6: the segment $\overline{\Gamma X}$ corresponds to the line $0 \leq k_{x} \leq \pi / p, k_{y}=0$, the segment $\overline{\mathrm{XM}}$ to $k_{x}=\pi / p, 0 \leq k_{y} \leq \pi / p$, and $\overline{\mathrm{M} \Gamma}$ to $0 \leq k_{x} \leq \pi / p, k_{y}=k_{x}$ (where $p=p_{x}=p_{y}$ ). The centrally symmetric unit cell is shown with dotted lines at the top left of Fig. 6. However, the unit cell with solid lines shown at the bottom left of Fig. 6 was used to derive the dispersion diagram. This choice avoids the presence of scatterers at the boundaries of the unit cell where equivalent voltages and currents are defined. A good match between the curves validates our results for all the considered propagation directions.

\section{B. Parameter Study on Reducibility Condition}

Here, we perform a parameter study on the structure of fig. 5 (a). We consider the propagation of the Bloch waves to be in the $x$ direction, and we discuss the number of modes needed for convergence of the first two Bloch modes

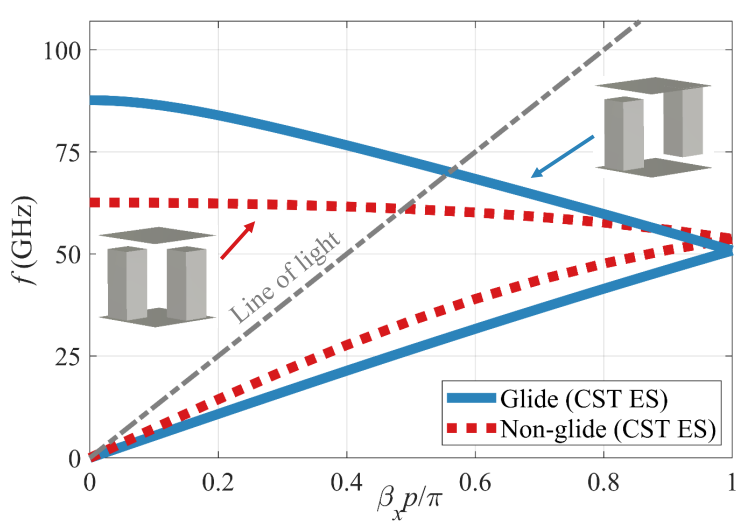

(a)

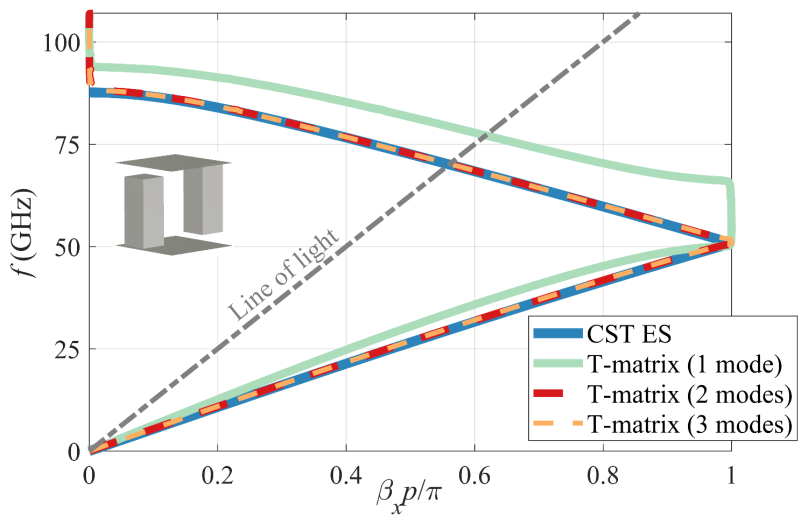

(b)

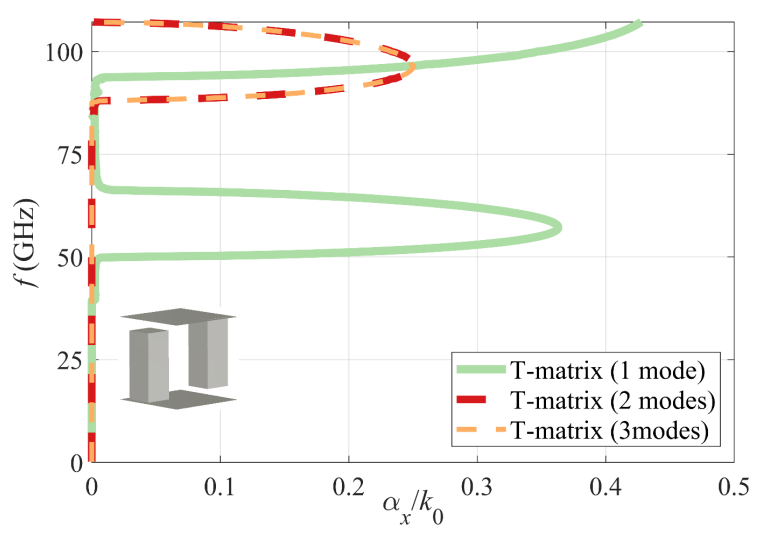

(c)

Fig. 7. Dispersion diagram assuming a propagation along the $x$ direction (i.e., $k_{y}=0$ ) for the glide-symmetric structure in Fig. 5(a). Geometrical parameters: $p_{x}=p_{y}=1.2 \mathrm{~mm}, d=0.5 \mathrm{~mm}, h_{1}=1 \mathrm{~mm}$ and $h=1.15$ $\mathrm{mm}$.

(e.g., from 0 to $100 \mathrm{GHz}$ ) using the transmission-matrix method. Fig. 9 (a) shows the required number of port modes for different periods while all the other parameters are kept constant. One can observe that as the period increases, fewer modes are needed for the convergence. This could be predicted since as the period increases, the corrugations are further away from each other, causing weaker couplings between them. Fig. 9 (b) and fig. 9 (c) show similar plots for different pin widths $(d)$ and different pin heights $\left(h_{1}\right)$. An increase in $h_{1}$ or in $d$, increases the number of modes required for the 


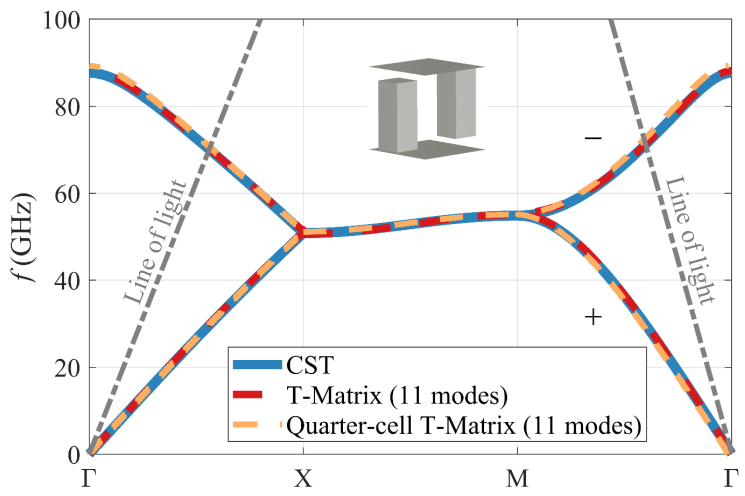

Fig. 8. Full dispersion diagram of the structure in Fig. 5(a). Geometrical parameters: $p_{x}=p_{y}=1.2 \mathrm{~mm}, d=0.5 \mathrm{~mm}, h_{1}=1 \mathrm{~mm}$ and $h=1.15$ $\mathrm{mm}$.

method's convergence. This can be explained by the fact that the increase of these values causes a closer proximity of the corrugations, thus making more waveguide modes significant.

The requirement of higher-order modes is partially related to the cut-off frequencies of the waveguide modes. In fact, as the number of modes increases, the higher-order modes become significant according to the order of their cutoff frequencies. However, modes can be necessary even if under cutoff. For instance, the first two Floquet modes of the 2D glide-symmetric structure of Fig. 5 (a) in Fig. 7 are both below the cut-off frequencies of all TM and TE modes of the exciting waveguide. Nevertheless, 11 modes are still needed to calculate the dispersion diagram.

The study of the convergence can be performed by running different simulations for increasing $N$, until the convergence is met. However, one can eliminate this step by knowing in advance how many modes are needed from the beginning. Our experiments show that the magnitude of the scattering parameters can be a good predictor of the number of significant modes. Significant modes are those for which at least one of the scattering parameters has a magnitude higher than a certain threshold (in our numerical results, a threshold of $-10 \mathrm{~dB}$ is sufficient).

\section{2-D Glide Conditions on the Boundaries of a Quarter of a Non-Minimal Unit Cell}

Section III discussed the exploitation of higher-order symmetries of the 1-D glide-symmetric structures in order to simplify the calculations for obtaining the dispersion relation. Here, we will take a similar approach to come up with similar equations for a quarter of a 2-D unit cell. First, we need to define a proper quarter-cell unit that has the proper symmetric characteristics to utilize. The generalized Floquet theorem for 2-D glide symmetric structures, not treated in [8], reads

$$
\begin{aligned}
& G_{m p_{x} \hat{\boldsymbol{x}}, n p_{y} \hat{\boldsymbol{y}}}[\boldsymbol{E}(x, z)] \\
& =\boldsymbol{E}\left(x+m p_{x} / 2, y+n p_{y} / 2,-z\right) \\
& = \pm e^{-j\left(m k_{x} p_{x} / 2+n k_{y} p_{y} / 2\right)} \boldsymbol{E}(x, y, z)
\end{aligned}
$$

where $m, n$ are odd integers. The factor \pm in the last term of (17) allows to recover the translation property (13) if

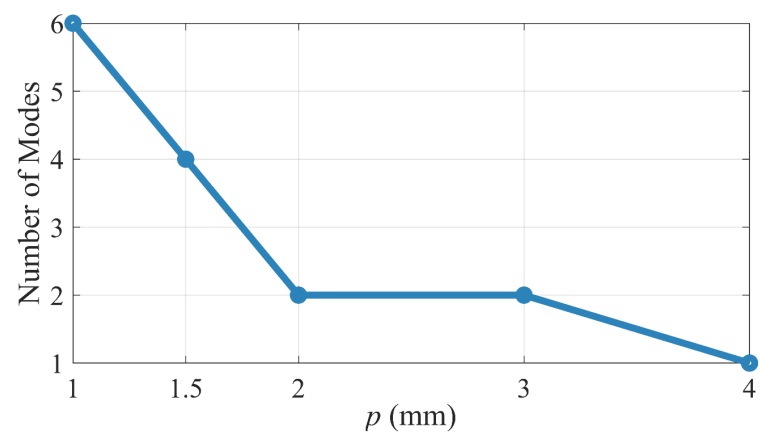

(a)

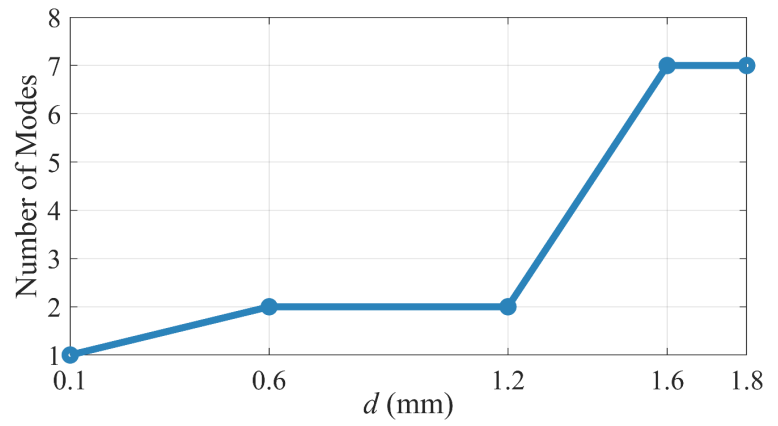

(b)

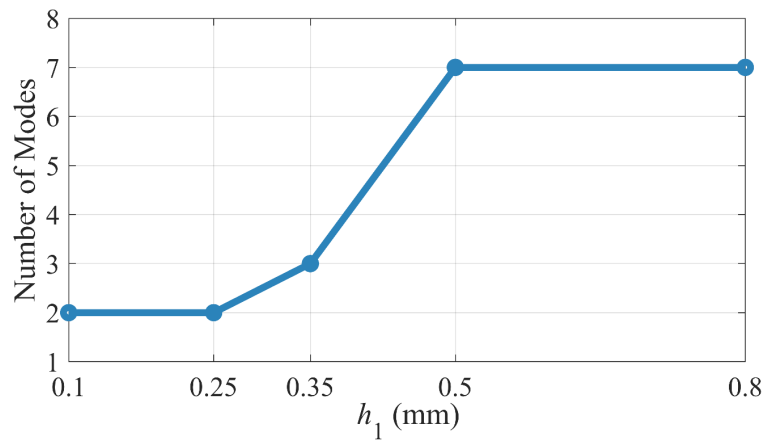

(c)

Fig. 9. Number of modes needed for convergence of the T-matrix method: (a) Versus period $p\left(h_{1}=0.5 \mathrm{~mm}, h=1.15 \mathrm{~mm}\right.$ and $\left.d=0.1 \mathrm{~mm}\right)$. (b) Versus pin width $d\left(h_{1}=0.5 \mathrm{~mm}, h=1.15 \mathrm{~mm}\right.$ and $\left.p=4 \mathrm{~mm}\right)$. (c) Versus pin height $h_{1}(p=4 \mathrm{~mm}, h=1.15 \mathrm{~mm}$ and $d=0.1 \mathrm{~mm})$.

two glides operations are applied. Note that in this case $m \neq 0, n \neq 0$, and the boundaries of the minimal unit cell defined by (14) cannot be obtained. We choose to enforce (17) on the boundaries defined by the two independent conditions $m=1, n=1$ and $m=1, n=-1$ :

$$
\begin{aligned}
& \boldsymbol{E}\left(x+\frac{p_{x}}{2}, y+\frac{p_{y}}{2},-z\right)= \pm e^{-j\left(k_{x} \frac{p_{x}}{2}+k_{y} \frac{p_{y}}{2}\right)} \boldsymbol{E}(x, y, z) \\
& \boldsymbol{E}\left(x+\frac{p_{x}}{2}, y-\frac{p_{y}}{2},-z\right)= \pm e^{-j\left(k_{x} \frac{p_{x}}{2}-k_{y} \frac{p_{y}}{2}\right)} \boldsymbol{E}(x, y, z)
\end{aligned}
$$

These boundaries define a quarter of the non-minimal cell with rotated periodic directions shown at the right side of Fig. 6 (dashed lines). In order to recover the plus sign in (13) after the two glide operations in (18) are applied sequentially, the same \pm signs must be chosen in both equations. Notice that if either $k_{x}$ or $k_{y}$ is fixed, the sign choice is arbitrary as in the 1-D case, as long as the same sign is chosen. A change in both 
equations changes the harmonic of the fixed wavenumber. On the contrary, the choice is important if propagation along a certain skew direction is considered and the condition $k_{y}=$ $c k_{x}$ is enforced, with $c$ a non-zero constant. In this case, the choice of the (same) sign in (18) depends on the mode to be found (more specifically, on its behavior within each unit cell). This will be detailed below for the numerical example shown.

Conditions (18) can be transformed into the following finitedimensional problem whose domain is restricted to a quarter of non-minimal unit cell whose transmission matrix is $\boldsymbol{T}_{1 / 4}$ :

$$
\underline{\boldsymbol{T}}_{1 / 4} \cdot\left[\begin{array}{l}
\boldsymbol{V}_{1} \\
\boldsymbol{V}_{2} \\
\boldsymbol{I}_{1} \\
\boldsymbol{I}_{2}
\end{array}\right]= \pm\left[\begin{array}{ll}
\frac{\boldsymbol{Q}}{\mathbf{0}} & \underline{\mathbf{0}}
\end{array}\right] \cdot\left[\begin{array}{l}
e^{-j\left(k_{x} \frac{p_{x}}{2}+k_{y} \frac{p_{y}}{2}\right)} \boldsymbol{V}_{1} \\
e^{-j\left(k_{x} \frac{p_{x}}{2}-k_{y} \frac{p_{y}}{2}\right)} \boldsymbol{V}_{2} \\
e^{-j\left(k_{x} \frac{p_{x}}{2}+k_{y} \frac{p_{y}}{2}\right)} \boldsymbol{I}_{1} \\
e^{-j\left(k_{x} \frac{p_{x}}{2}-k_{y} \frac{p_{y}}{2}\right)} \boldsymbol{I}_{2}
\end{array}\right]
$$

where the \pm sign, being the same in the two boundaries, can be brought outside the vectors in the right terms. The matrix $\underline{Q}$ is defined as in (9) with respect to the parity of the vertical components of the modal electric fields $e_{z}$, used to define the equivalent voltages.

Fig. 8 also depicts the full dispersion diagram of the irreducible structure of Fig. 5 computed from the $T$ matrix of a quarter of the unit cell using (19). The plot shows that with 11 modes, a convergence to the correct solution is reached. As an example, the first four relevant modes are those of a rectangular waveguide with PMC lateral walls. They are the TEM, $\mathrm{TM}_{01}, \mathrm{TE}_{10}$ and $\mathrm{TE}_{11}$ modes. The TEM and $\mathrm{TE}_{10}$ have even symmetry with respect to $z$, so the sign factors $q^{(1)}$ and $q^{(3)}$ are chosen equal to +1 . In contrast, $\mathrm{TM}_{01}$ and $\mathrm{TE}_{11}$ have odd symmetry in the $z$ direction and a $q^{(i)}$ factor of -1 is adopted for these modes. The same procedure applies to the higher modes.

Close to each curve, we show the sign used in (19) to recover the mode. As stated previously, in the segments $\Gamma-\mathrm{X}$ and $\mathrm{X}-\mathrm{M}$ the choice of the sign is arbitrary since $k_{y}=0$ and $k_{x}=\pi / p_{x}$ is fixed, respectively. In the segment $\mathrm{M}-\Gamma$ $k_{x}=k_{y}$, and the sign used is shown close to each curve.

\section{Computational Time}

To have a comparison between the computational time of the multimodal transmission-matrix method and the CST eigensolver, we consider the 2-D glide-symmetric example discussed in Sec. IV. We apply the two methods and compare the execution time for each of them. In this comparison, we used a computer with $128 \mathrm{~GB}$ of RAM and an Intel(R) Xeon(R) CPU with 6 cores and a base frequency of $3.60 \mathrm{GHz}$ for its CPU cores. First, it is necessary to note that it is not very straightforward to compare the time cost of these two methods due to the difference in their nature. For instance, the CST eigensolver performs a sweep of the phase constant, whereas the transmission-matrix method does a sweep on the frequency.

For the comparison performed, we used 74 points on the dispersion diagram in both methods. The execution time in CST eigensolver was 402 seconds. Next, we applied the transmission-matrix method in two steps. In the first step,
TABLE I

COMPUTATIONAL TIME FOR SOLVING THE EIGENVALUE PROBLEM IN (19)

\begin{tabular}{|c|c|c|c|}
\hline $\begin{array}{c}\text { Number of } \\
\text { modes } \\
(N)\end{array}$ & Time (seconds) & $\begin{array}{c}\text { Number of } \\
\text { modes } \\
(N)\end{array}$ & Time (seconds) \\
\hline 1 & 0.04 & 7 & 0.39 \\
2 & 0.07 & 8 & 0.49 \\
3 & 0.07 & 9 & 0.52 \\
4 & 0.08 & 10 & 0.64 \\
5 & 0.11 & 11 & 0.88 \\
6 & 0.16 & 12 & 1.13 \\
\hline
\end{tabular}

we used the CST Frequency solver to extract the scattering parameters for the quarter of a unit cell used in Sec. IV-C. For this matter, the frequency range of $0-90 \mathrm{GHz}$ was used and four waveguide ports were applied with 12 modes at each port. This gave us the multimodal scattering matrix for $N \leq 12$. The execution time for the first step was 82 seconds. Then, we calculated the transmission matrix from the CST scattering parameters using a simple MATLAB code [38], whose execution time (around $10 \mathrm{~ms}$ ) is negligible. In the second step, we used the algorithm in [39] to find the complex roots of the eigenvalue problem in (19), again in MATLAB. The execution time of the second step is given in Table I, and it is clearly much faster than the full-wave simulation. In fact, calculation of all the first 11 cases (11 modes were needed for convergence) takes less than 4 seconds in total. As a result, the standard CST eigenvalue solver (solving for a full cell) required $402 \mathrm{~s}$, while the transmission matrix method (solving for a quarter of cell) with 12 modes required $82+1.13 \mathrm{~s}$ with 12 modes at each port.

\section{CONCLUSION}

The connection between glide-symmetric and non-glide symmetric structure with reduced period has been discussed in this paper. Reducible and irreducible glide structures have been defined, depending whether the effect of glide symmetry is a simple division by two of the period or not, respectively. A condition to distinguish between these structures is given in terms of relevance of higher-order modal interactions among periodic scatterers.

A new formulation in terms of glide-symmetric boundary conditions explicitly shows the different modal interactions due to mirrored scatterers and confirm the relevance of higherorder modes for irreducible structures.

All the formulations have been derived from eigenvalue problems related to relevant symmetry operators, transformed into finite-dimensional problems with a multimodal transmission matrix approach. The results have been presented and validated with commercial software, both in 1-D lines and in 2-D surfaces. In this last case, the choice of the computational domain as a quarter of a rotated non-minimal unit cell is motivated. All methods presented can compute both phase and attenuation constants of Bloch modes, for possible application to glide-symmetric leaky-waves.

\section{REFERENCES}

[1] D. M. Pozar, "Microwave engineering." John Wiley and Sons, Inc., 2011, ch. 8: Microwave Filters. 
[2] J. D. Joannopoulos and S. G. Johnson, Photonic Crystals: Molding the Flow of Light. Princeton University Press, 2008.

[3] G. V. Eleftheriades and K. G. Balmain, Negative-Refraction Metamaterials: Fundamental Principles and Applications. Wiley-IEEE Press, 2005.

[4] O. Dahlberg, R. C. Mitchell-Thomas, and O. Quevedo-Teruel, "Reducing the dispersion of periodic structures with twist and polar glide symmetries," Scientific Reports, vol. 7, Art. no. 10136, 2017.

[5] F. Ghasemifard, M. Norgren, and O. Quevedo-Teruel, "Twist and polar glide symmetries: an additional degree of freedom to control the propagation characteristics of periodic structures," Scientific Reports, vol. 8, no. 1, p. 11266, 2018. [Online]. Available: https://doi.org/10.1038/s41598-018-29565-6

[6] O. Quevedo-Teruel, O. Dahlberg, and G. Valerio, "Propagation in waveguides with transversal twist-symmetric holey metallic plates," IEEE Microwave and Wireless Components Letters, 2018.

[7] P. Padilla, L. F. Herrn, A. Tamayo-Domnguez, J. F. Valenzuela-Valds, and O. Quevedo-Teruel, "Glide symmetry to prevent the lowest stopband of printed corrugated transmission lines," IEEE Microwave and Wireless Components Letters, vol. 28, no. 9, pp. 750-752, Sept 2018.

[8] A. Hessel, M. H. Chen, R. C. M. Li, and A. A. Oliner, "Propagation in periodically loaded waveguides with higher symmetries," Proceedings of the IEEE, vol. 61, no. 2, pp. 183-195, Feb. 1973.

[9] P. J. Crepeau and P. R. McIsaac, "Consequences of symmetry in periodic structures," Proceedings of the IEEE, vol. 52, no. 1, pp. 33-43, Jan 1964.

[10] R. Mittra and S. Laxpati, "Propagation in a wave guide with glide reflection symmetry," Canadian Journal of Physics, vol. 43, no. 2, pp. 353-372, 1965. [Online]. Available: https://doi.org/10.1139/p65-032

[11] R. Kieburtz and J. Impagliazzo, "Multimode propagation on radiating traveling-wave structures with glide-symmetric excitation," IEEE Transactions on Antennas and Propagation, vol. 18, no. 1, pp. 3-7, Jan 1970.

[12] R. Quesada, D. Martín-Cano, F. J. García-Vidal, and J. Bravo-Abad, "Deep-subwavelength negative-index waveguiding enabled by coupled conformal surface plasmons," Opt. Lett., vol. 39, no. 10, pp. 2990-2993, May 2014

[13] J. J. Wu, C.-J. Wu, D. J. Hou, K. Liu, and T.-J. Yang, "Propagation of low-frequency spoof surface plasmon polaritons in a bilateral crossmetal diaphragm channel waveguide in the absence of bandgap," IEEE Photonics Journal, vol. 7, no. 1, pp. 1-8, Feb. 2015.

[14] D. Cavallo and C. Felita, "Analytical formulas for artificial dielectrics with non-aligned layers," IEEE Transactions on Antennas and Propagation, vol. PP, no. 99, pp. 1-1, 2017.

[15] T. Chang, J. U. Kim, S. K. Kang, H. Kim, D. K. Kim, Y.-H. Lee, and J. Shin, "Broadband giant-refractive-index material based on mesoscopic space-filling curves," Nature Materials, vol. 7, pp. 772-775, Aug 2016. [Online]. Available: https://www.nature.com/articles/ncomms12661

[16] D. Jia, Y. He, N. Ding, J. Zhou, B. Du, and W. Zhang, "Beam-steering flat lens antenna based on multilayer gradient index metamaterials," IEEE Antennas and Wireless Propagation Letters, vol. 17, no. 8, pp. 1510-1514, Aug 2018.

[17] O. Quevedo-Teruel, M. Ebrahimpouri, and M. N. M. Kehn, "Ultrawideband metasurface lenses based on off-shifted opposite layers," IEEE Antennas and Wireless Propagation Letters, vol. 15, pp. 484-487, Dec. 2016.

[18] O. Quevedo-Teruel, J. Miao, M. Mattsson, A. Algaba-Brazalez, M. Johansson, and L. Manholm, "Glide-symmetric fully metallic Luneburg lens for 5G communications at Ka-band," IEEE Antennas and Wireless Propagation Letters, vol. 17, no. 9, pp. 1588-1592, Sept 2018.

[19] O. Quevedo-Teruel, M. Ebrahimpouri, and F. Ghasemifard, "Lens antennas for 5G communications systems," IEEE Communications Magazine, vol. 56, no. 7, pp. 36-41, JULY 2018.

[20] M. Ebrahimpouri, A. A. Brazalez, L. Manholm, and O. Quevedo-Teruel, "Using glide-symmetric holes to reduce leakage between waveguide flanges," IEEE Microwave and Wireless Components Letters, vol. 28 , no. 6, pp. 473-475, June 2018.

[21] M. Ebrahimpouri, E. Rajo-Iglesias, Z. Sipus, and O. Quevedo-Teruel, "Cost-effective gap waveguide technology based on glide-symmetric holey EBG structures," IEEE Transactions on Microwave Theory and Techniques, vol. 66, no. 2, pp. 927-934, Feb 2018.

[22] E. Rajo-Iglesias, M. Ebrahimpouri, and O. Quevedo-Teruel, "Wideband phase shifter in groove gap waveguide technology implemented with glide-symmetric holey EBG," IEEE Microwave and Wireless Components Letters, vol. 28, no. 6, pp. 476-478, June 2018.

[23] G. Valerio, F. Ghasemifard, Z. Sipus, and O. Quevedo-Teruel, "Glidesymmetric all-metal holey metasurfaces for low-dispersive artificial materials: Modeling and properties," IEEE Transactions on Microwave Theory and Techniques, vol. 66, no. 7, pp. 3210-3223, July 2018.
[24] F. Ghasemifard, M. Norgren, O. Quevedo-Teruel, and G. Valerio, "Analyzing glide-symmetric holey metasurfaces using a generalized floquet theorem," IEEE Access, vol. 6, pp. 71 743-71 750, 2018.

[25] F. Ghasemifard, M. Norgren, and O. Quevedo-Teruel, "Dispersion analysis of 2-D glide-symmetric corrugated metasurfaces using modematching technique," IEEE Microwave and Wireless Components Letters, vol. 28, no. 1, pp. 1-3, Jan 2018.

[26] G. Valerio, Z. Sipus, A. Grbic, and O. Quevedo-Teruel, "Accurate equivalent-circuit descriptions of thin glide-symmetric corrugated metasurfaces," IEEE Transactions on Antennas and Propagation, vol. 65, no. 5, pp. 2695-2700, May 2017.

[27] Q. Chen, F. Ghasemifard, G. Valerio, and O. Quevedo-Teruel, "Modeling and dispersion analysis of coaxial lines with higher symmetries," IEEE Transactions on Microwave Theory and Techniques, no. 99, pp. 1-8, 2018.

[28] N. Marcuvitz, Waveguide Handbook. Isha Books, 2013.

[29] F. Mesa, R. Rodríguez-Berral, and F. Medina, "On the computation of the dispersion diagram of symmetric one-dimensionally periodic structures," Symmetry, vol. 10, no. 8, 2018. [Online]. Available: http://www.mdpi.com/2073-8994/10/8/307

[30] M. A. Eberspcher and T. F. Eibert, "Dispersion analysis of complex periodic structures by full-wave solution of even-odd-mode excitation problems for single unit cells," IEEE Transactions on Antennas and Propagation, vol. 61, no. 12, pp. 6075-6083, Dec 2013.

[31] R. Collin and F. Zucker, Antenna Theory, ser. Inter-University Electronics Series. McGraw-Hill, 1969, no. pt. 2.

[32] J. Volakis, Antenna Engineering Handbook, Fourth Edition. McGraw-Hill Companies,Incorporated, 2007. [Online]. Available: https://books.google.it/books?id=bmdFAAAAYAAJ

[33] G. Valerio, S. Paulotto, P. Baccarelli, P. Burghignoli, and A. Galli, "Accurate Bloch analysis of 1-D periodic lines through the simulation of truncated structures," IEEE Transactions on Antennas and Propagation, vol. 59, no. 6, pp. 2188-2195, Jun. 2011.

[34] "CST Microwave Studio," http://www.cst.com/, version: 2016.

[35] C. Molero, R. Rodríguez-Berral, F. Mesa, and F. Medina, "Analytical circuit model for 1-D periodic T-shaped corrugated surfaces," IEEE Transactions on Antennas and Propagation, vol. 62, no. 2, pp. 794 803, February 2014.

[36] S. Monni, G. Gerini, A. Neto, and A. G. Tijhuis, "Multimode equivalent networks for the design and analysis of frequency selective surfaces," IEEE Transactions on Antennas and Propagation, vol. 55, no. 10, pp. 2824-2835, Oct 2007.

[37] R. Rodriguez-Berral, F. Mesa, and F. Medina, "Analytical multimodal network approach for 2-D arrays of planar patches/apertures embedded in a layered medium," IEEE Transactions on Antennas and Propagation, vol. 63, no. 5, pp. 1969-1984, May 2015.

[38] MATLAB, version 9.3.0 (R2017b). Natick, Massachusetts: The MathWorks Inc., 2017.

[39] V. Galdi and I. M. Pinto, "A simple algorithm for accurate location of leaky-wave poles for grounded inhomogeneous dielectric slabs," Microwave and optical technology letters, vol. 24, no. 2, pp. 135-140, 2000.

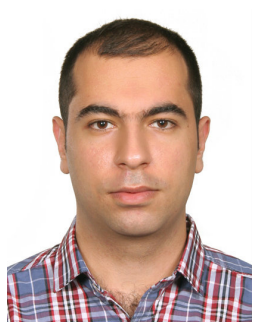

Mohammad Bagheriasl received the M.Sc. degree in fields and waves in 2016 from university of Tehran, Tehran, Iran. Since October 2017, he is a $\mathrm{Ph} . \mathrm{D}$. student in the Laboratoire d'Électronique et Électromagnétisme, at Sorbonne Université, Paris, France. His scientific interests involve small antenna design, characteristic mode theory and modeling and analysis of periodic structures with highersymmetries. 


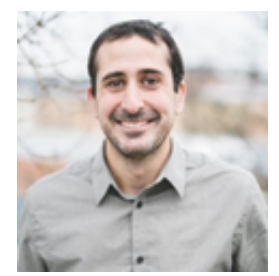

Oscar Quevedo-Teruel is a Senior Member of the IEEE. He received his degree in Telecommunication Engineering from Carlos III University of Madrid Spain in 2005, part of which was done at Chalmers University of Technology in Gothenburg, Sweden. $\mathrm{He}$ obtained his $\mathrm{PhD}$ from Carlos III University of Madrid in 2010 and was then invited as a postdoctoral researcher at the University of Delft (The Netherlands). From 2010-2011, Dr. QuevedoTeruel joined the Department of Theoretical Physics of Condensed Matter at Universidad Autonoma de Madrid as a research fellow, and went on to continue his postdoctoral research at Queen Mary University of London from 2011-2013.

In 2014, he joined the Electromagnetic Engineering Division, in the School of Electrical Engineering and Computer Science at KTH Royal Institute of Technology in Stockholm, Sweden where he is an Associate Professor and director of the Master Programme in Electromagnetics Fusion and Space Engineering. He is an Associate Editor of the IEEE Transactions on Antennas and Propagation since 2018, and he is the delegate of EurAAP for Sweden, Norway and Iceland for the period 2018-2020. He is a distinguished lecturer of the IEEE Antennas and Propagation Society for the period 2019-2021.

He has made scientific contributions to higher symmetries (glide and twist), transformation optics, lens antennas, metasurfaces, leaky wave antennas, multi-mode microstrip patch antennas and high impedance surfaces. He is the co-author of more than 70 papers in international journals, more than 120 at international conferences and has received approval on 2 patents.

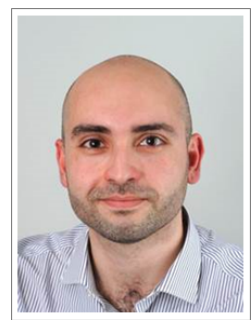

Guido Valerio (S'06-M'10-SM'18) received the M.S. degree (cum laude and honorable mention) in electronic engineering in 2005, and the $\mathrm{Ph} . \mathrm{D}$. degree in electromagnetics in 2009, from La Sapienza University, Rome, Italy. From February to August 2008 he was a Visiting Scholar at the University of Houston, TX, USA. From 2011 to 2014 , he was a researcher at the Institute d'Électronique et de Télécommunications de Rennes (IETR), France. Since September 2014 he is an Associate Professor in the Laboratoire d'Électronique et Électromagnétisme, at Sorbonne Université, Paris, France.

His scientific interests involve antenna design and numerical methods for wave propagation and scattering in complex structures; namely, periodic Greens function computation, modal properties of multilayered structures, full-wave methods for SIW, modeling of periodic structures with highersymmetries.

In 2008 Dr. Valerio was the recipient of the "Leopold B. Felsen Award for Excellence in Electrodynamics." In 2009 he was a finalist for the "Young Engineering Prize" at the European Microwave Conference. In 2010 he was the recipient of the Barzilai Prize for the best paper at the National Italian Congress of Electromagnetism (XVIII RiNEm). In 2014, he was the recipient of the RMTG Award for junior researchers presented at the IEEE Antennas and Propagation Society Symposium, Memphis, TN. In 2018, he was a coauthor of the best paper in Electromagnetic and Antenna theory at the 12th European Conference on Antennas and Propagation, London, U.K. 